\title{
The utility of routine surveillance screening with magnetic resonance imaging (MRI) to detect tumour recurrence in children with low-grade central nervous system (CNS) tumours: a systematic review
}

\author{
Simon P. Stevens ${ }^{1} \cdot$ Caroline Main $^{1} \cdot$ Simon Bailey $^{2} \cdot$ Barry Pizer $^{3} \cdot$ Martin English $^{5} \cdot$ Robert Phillips $^{6} \cdot$ Andrew Peet $^{4}$. \\ Shivaram Avula ${ }^{3} \cdot$ Sophie Wilne ${ }^{7} \cdot$ Keith Wheatley $^{1} \cdot$ Pamela R. Kearns $^{1,5} \cdot$ Jayne S. Wilson ${ }^{1}$
}

Received: 16 February 2018 / Accepted: 12 May 2018 / Published online: 9 June 2018

(c) The Author(s) 2018

\begin{abstract}
Background Magnetic resonance imaging (MRI) is routinely used as a surveillance tool to detect early asymptomatic tumour recurrence with a view to improving patient outcomes. This systematic review aimed to assess its utility in children with low-grade CNS tumours.

Methods Using standard systematic review methods, twelve databases were searched up to January 2017.

Results Seven retrospective case series studies $(\mathrm{n}=370$ patients) were included, with average follow-up ranging from 5.6 to 7 years. No randomised controlled trials (RCTs) were identified. Due to study heterogeneity only a descriptive synthesis could be undertaken. Imaging was most frequent in the first year post-surgery (with 2-4 scans) reducing to around half this frequency in year two and annually thereafter for the duration of follow-up. Diagnostic yield ranged from 0.25 to $2 \%$. Recurrence rates ranged from 5 to $41 \%$, with most recurrences asymptomatic (range 65-100\%). Collectively, 56\% of recurrences had occurred within the first year post-treatment ( $46 \%$ in the first 6 -months), $68 \%$ by year two and $90 \%$ by year five. Following recurrence, $90 \%$ of patients underwent treatment changes, mainly repeat surgery (72\%). Five-year OS ranged from 96 to $100 \%$, while five-year recurrence-free survival ranged from 67 to $100 \%$. None of the studies reported quality of life measures. Conclusion This systematic review highlights the paucity of evidence currently available to assess the utility of MRI surveillance despite it being routine clinical practice and costly to patients, their families and healthcare systems. This needs to be evaluated within the context of an RCT.
\end{abstract}

Keywords Systematic review $\cdot$ Magnetic resonance imaging (MRI) $\cdot$ Surveillance $\cdot$ Children $\cdot$ Central nervous system $($ CNS) tumours $\cdot$ Recurrence $\cdot$ Pilocytic astrocytoma $\cdot$ Low grade glioma

Electronic supplementary material The online version of this article (https://doi.org/10.1007/s11060-018-2901-x) contains supplementary material, which is available to authorized users.

Jayne S. Wilson

j.s.wilson.1@bham.ac.uk

1 Cancer Research UK Clinical Trials Unit (CRCTU), Institute of Cancer and Genomic Sciences, University of Birmingham, Birmingham, UK

2 Sir James Spence Institute of Child Health, Royal Victoria Infirmary, Newcastle upon Tyne, UK

3 Alder Hey Children's NHS Foundation Trust, Liverpool, UK

\section{Introduction}

Paediatric low-grade CNS tumours are an extremely diverse group of neoplasms. The likelihood of recurrence is largely a function of tumour type, but also varies according to tumour location as well as treatment regimens [1].

4 Institute of Cancer and Genomic Sciences, University of Birmingham, Birmingham, UK

5 Birmingham Women and Children's Hospital NHS Foundation Trust, Birmingham, UK

6 Centre for Reviews and Dissemination (CRD), University of York, York, UK

7 Queen's Medical Centre, Nottingham University Hospitals' NHS Trust, Nottingham, UK 
Surveillance neuroimaging is routinely used to detect recurrence in children with low-grade CNS tumours in the absence of clinical signs and symptoms. The rationale behind surveillance is that recurrence detected at a stage when there is less disease will be more responsive to treatment and this will result in improved outcomes for patients. The scheduling and imaging techniques employed, or surveillance protocols, are loosely based on the biological characteristics of the different CNS tumour types, taking into account the rate of tumour growth, location and patterns of local and metastatic recurrence [2, 3].

In recent years, magnetic resonance imaging (MRI) has replaced computed tomography (CT) as the dominant surveillance neuroimaging modality. Its greater imaging resolution and multi-planar capability account for its superior diagnostic utility, particularly with respect to soft tissue neoplasms such as CNS tumours [4]. However, despite being standard practice, there have been no systematic reviews assessing surveillance MRI in this patient group.

The aims of this systematic review were to:

1. Assess the utility of surveillance MRI to detect early tumour recurrence in children with no new, stable or improved neurological signs or symptoms with lowgrade CNS tumours compared to the use of non-routine imaging undertaken on presentation with disease signs or symptoms and whether this results in improved clinical outcomes for patients and their families;

2. Evaluate the effects of varying MRI screening intervals across tumour types and determine the optimum length of time for screening post-initial diagnosis;

3. Identify gaps and methodological weaknesses in the current evidence base to inform the design of future studies.

\section{Methods}

This review is part of a series of systematic reviews looking at treatments for paediatric CNS tumours. The project included public and patient involvement (PPI), consisting of the parents of children with CNS tumours who expressed a particular interest in this review question, which was pivotal in our decision to undertake the current review.

Standard systematic review methodology aimed at minimising bias was employed and reporting followed the Preferred Reporting Items for Systematic Reviews and MetaAnalyses (PRISMA) guidelines [5]. A detailed account of the methodology employed in this review can be found in the published protocol, which is also registered with PROSPERO (CRD42016036802) [6].

\section{Search strategy}

Searches for published studies from 1985 to January 2017 were undertaken in a number of databases, including MEDLINE, EMBASE and the Cochrane Library. No language/ publication restrictions or study design filters were applied (see Online Resource 1 for search strategy and databases searched). Reference lists of included studies were citationchecked and experts in the field consulted for published studies not retrieved by the electronic searches.

\section{Study selection}

The following inclusion and exclusion criteria were applied: Population Children and young adults (age up to 25 years) with diagnoses of any type of low grade (i.e. WHO grade I and II) CNS tumour who had either no new, stable or improved neurological signs or symptoms at the time of study recruitment.

Intervention Routine interval follow-up MRI scans conducted at any screening interval determined within the primary study. Studies reporting CT scans were excluded.

Outcomes Outcome measures included recurrence rates (by study, tumour type, location and extent of resection), diagnostic yield of imaging, timing of recurrence, change in patient management post-recurrence, overall survival (OS), surrogate survival measures (i.e. recurrence-free survival (RFS) and progression-free survival (PFS)) and quality of life.

Patients were deemed to have experienced a recurrence if, after undergoing complete surgical removal of their primary tumour [i.e. achieving a gross total resection (GTR)], evidence of tumour was captured on a subsequent MRI scan. Patients were deemed to have experienced progressive disease if, after undergoing incomplete surgical removal of their primary tumour [i.e. achieving a sub-total resection (STR)], evidence of a significant increase in the size of the tumour was captured on a subsequent MRI scan. However, for the purposes of this paper, we use the term 'recurrence' to cover both recurrence in GTR and progression in STR patients.

All of the studies in this review reported patient outcomes in terms of whether or not patients were asymptomatic at recurrence. However, children undergoing surveillance imaging for detection of recurrent disease will often be asymptomatic from the recurrence but may have some neurologic sequelae from their tumour and/or its treatment. This was the reason for characterising patients as having either no new, stable or improved neurological signs or symptoms.

Study designs Randomised controlled trials (RCTs) and non-randomised comparative studies were initially sought. 
However, as no such studies were identified the review was extended to include observational studies, such as case series studies. Single case reports, letters or editorials were excluded.

Study selection was undertaken by two independent reviewers. Citations marked for inclusion on the basis of title and abstract underwent full text assessment. Disagreements were resolved by discussion (see Online Resource 2 for details of excluded studies).

\section{Data extraction and risk of bias assessment}

Data was extracted by one reviewer and checked by a second and recorded on a standardised pro-forma (see Online Resource 3). Risk of bias was conducted by two reviewers and assessed at the study level using a six-point tool devised by the Centre for Reviews and Dissemination (York; CRD) specifically designed to assess bias in case series studies [7].

\section{Statistical analysis}

Due to the design of the studies included in the review and the heterogeneity of outcomes reported, only a descriptive analysis of the data was possible.

\section{Results}

\section{Quantity of the research}

From the electronic database searches, 28 publications were considered potentially relevant to this review, with an additional 13 identified from citation checking. On full text examination, 34 publications did not meet the inclusion criteria including 14 surveillance imaging studies which employed both CT and MRI but failed to report results separately for MRI. No RCTs or prospective comparative studies were identified. Seven retrospective case series were included in the review [8-14] (see Fig. 1 for the PRISMA flow diagram).

\section{Quality of the research}

All seven studies were undertaken at single centre institutions. Study samples were small but appeared to be representative of the relevant population, i.e. children with low-grade CNS tumours undergoing surveillance imaging using MRI to detect recurrence. In all studies, inclusion and exclusion criteria for each study were explicitly stated. Generally, participants were at a similar time point in the course of their disease in that all had undergone resection of their primary tumour, whether a GTR or STR. Definitions of GTR and STR were provided although definitions varied between studies (see Online Resource 4 for definitions table). No studies containing STR patients reported the size/extent of residual tumour. Duration of follow-up was reported in five studies [9, 11-14]. Assessment of outcomes using objective criteria was variable. The definition for radiographic recurrence was provided in all but two studies $[10,12]$ but again definitions varied between studies. All but one study [13] reported details of the change in patient management/ treatment post-recurrence. Definitions of survival outcome measures were provided in only half of the studies reporting survival, with one study failing to define OS [8] and one RFS [12] (see Online Resource 5 for quality assessment table).

\section{Description of included studies}

The seven studies were published between 2001 and 2016 with six published since 2009 [9-14]. Five studies were undertaken in the USA [8, 9, 11-13], one in Canada [14] and one in Australia [10]. The studies (overall $n=469$ patients) were comprised of six low grade tumour studies [9-14] and one with a mix of low and high-grade tumours [8]. The total number of patients in the low-grade studies was 357 with a mean sample size of 60 . Mean age at diagnosis was 7.5 years, $48 \%$ of patients were male and $75 \%$ of tumours were located in the posterior fossa. Median followup ranged from 5.6 to 7 years. Three studies $[9,11,13]$ explicitly excluded children with Neurofibromatosis Type 1 , with the remaining studies providing no details of the NF1 status of their study populations. At the commencement of surveillance imaging, none of the patients had relapsed disease (See Table 1).

The six low-grade tumour studies included patients with the following tumour types: pilocytic astrocytoma $(n=297)$, ganglioglioma $(n=22)$, dysembryoplastic neuroepithelial tumour (DNET) $(n=6)$, glioneuronal tumours $(n=1)$, nonpilocytic astrocytoma/diffuse fibrillary astrocytoma (DFA) $(n=18)$, optic glioma $(n=7)$, oligodendroglioma $(n=3)$ and 'other' astrocytoma $(\mathrm{n}=3)$.

\section{Korones [8]}

This was the only mixed grade tumour study but it also differed from the other studies in that it did not provide a breakdown of patients at the beginning of the study, instead reporting only the number of recurrences by tumour type. Thirteen LGG patients recurred (11 astrocytomas and two gangliogliomas). However, as the number of non-recurrent LGG patients was not reported, recurrence rates were not calculable and therefore data from this study is not comparable with that from the other studies. Of the 13 recurrences, ten patients (nine astrocytoma and one ganglioglioma) 


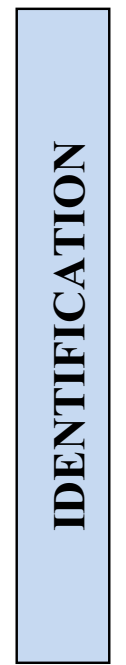

Records identified through database searching for all topics $(\mathrm{N}=10,072)$
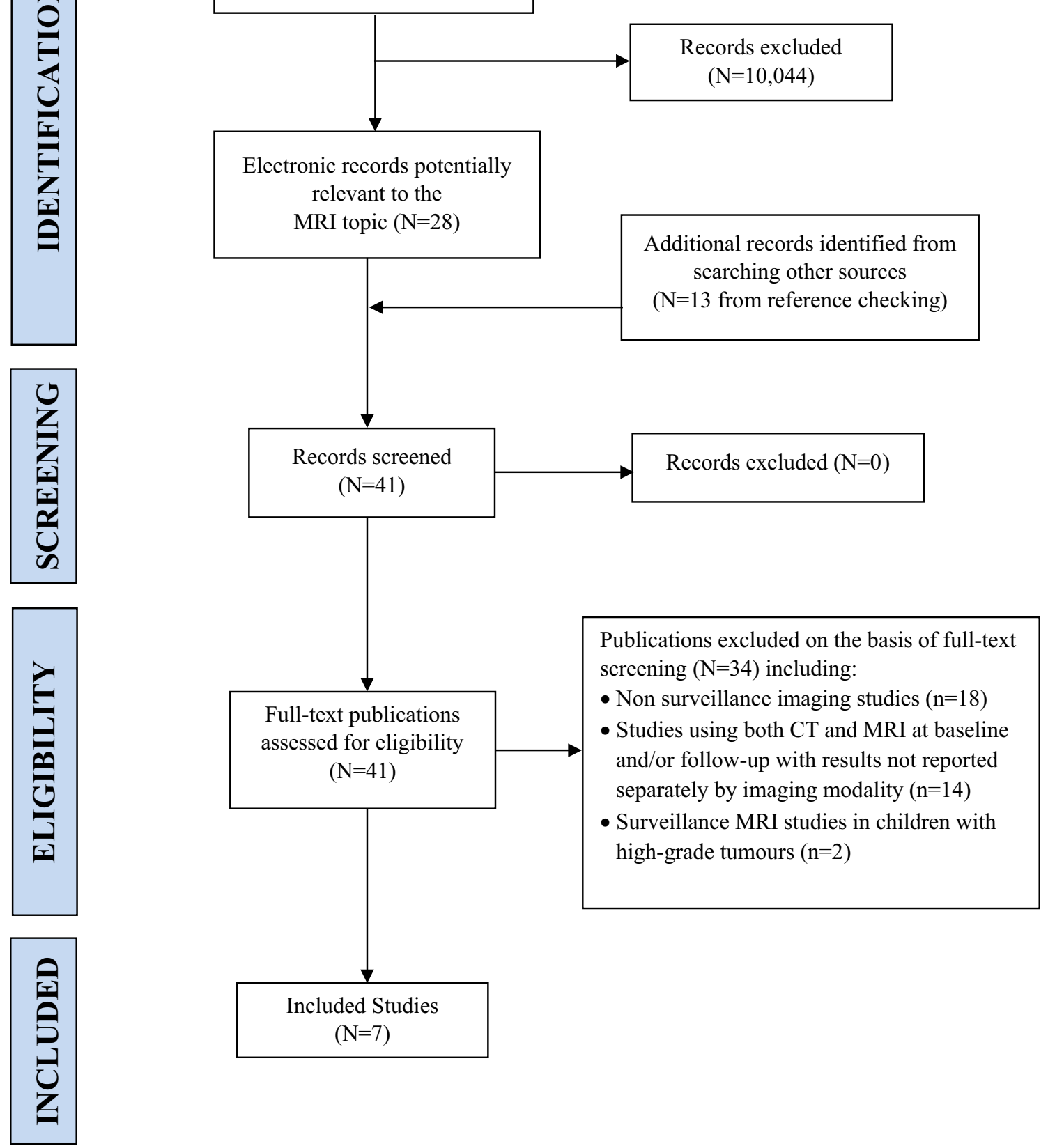

Fig. 1 PRISMA diagram of flow of studies through the selection process

were asymptomatic at recurrence giving a diagnostic yield of scanning of $3.7 \%$ (or one recurrence detected every 27 scans). Recurrent patients were scanned with a frequency of one scan every 5.3 months. Median time to recurrence was 2.3 years and OS was $100 \%$. Neither outcome was reported by tumour type.

\section{MRI protocols}

Four studies reported details regarding MRI scanners used, image sequences, weighting and contrast enhancement $[9$, 11, 13, 14]. (For details, see Table 1). 


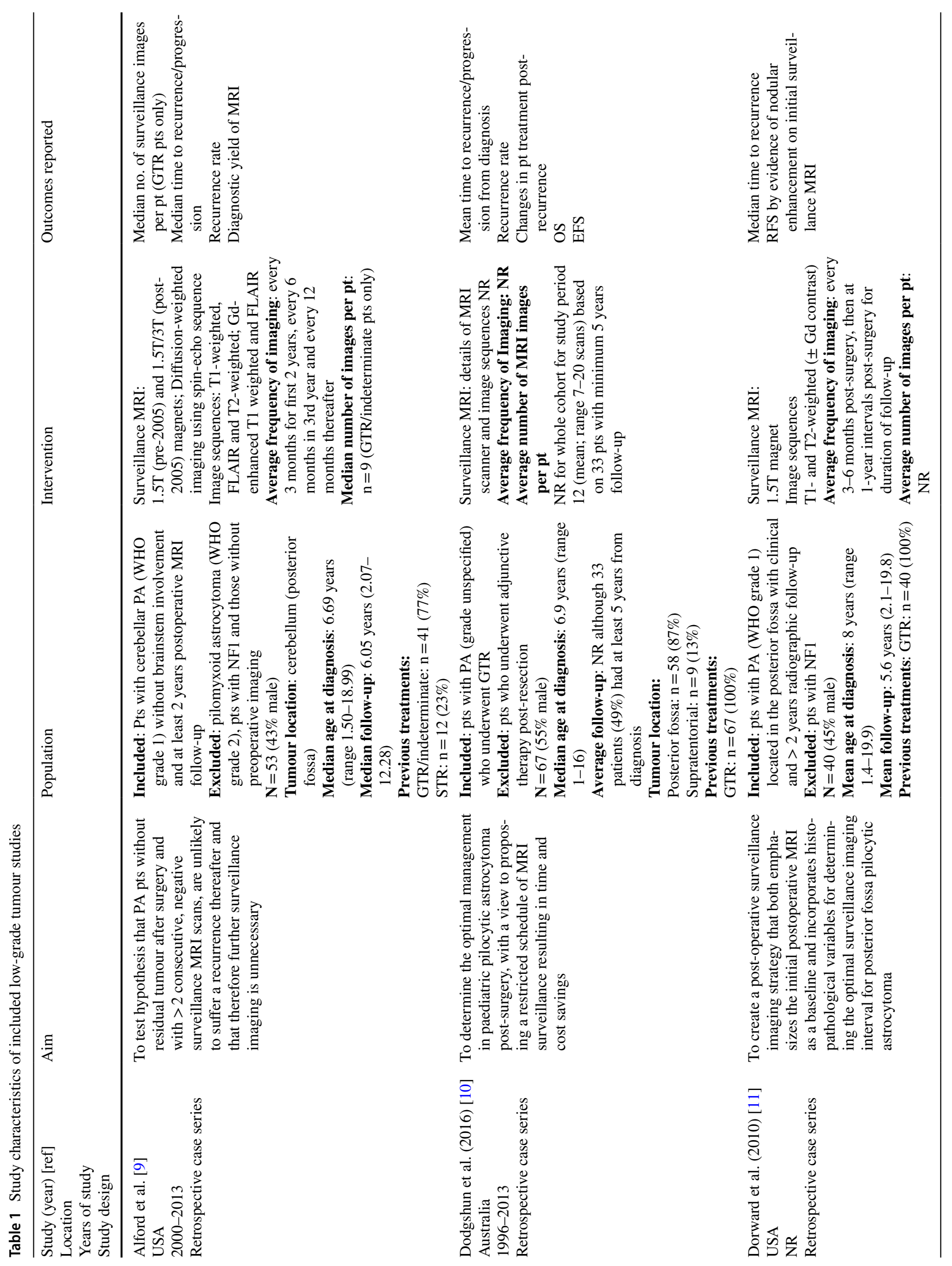




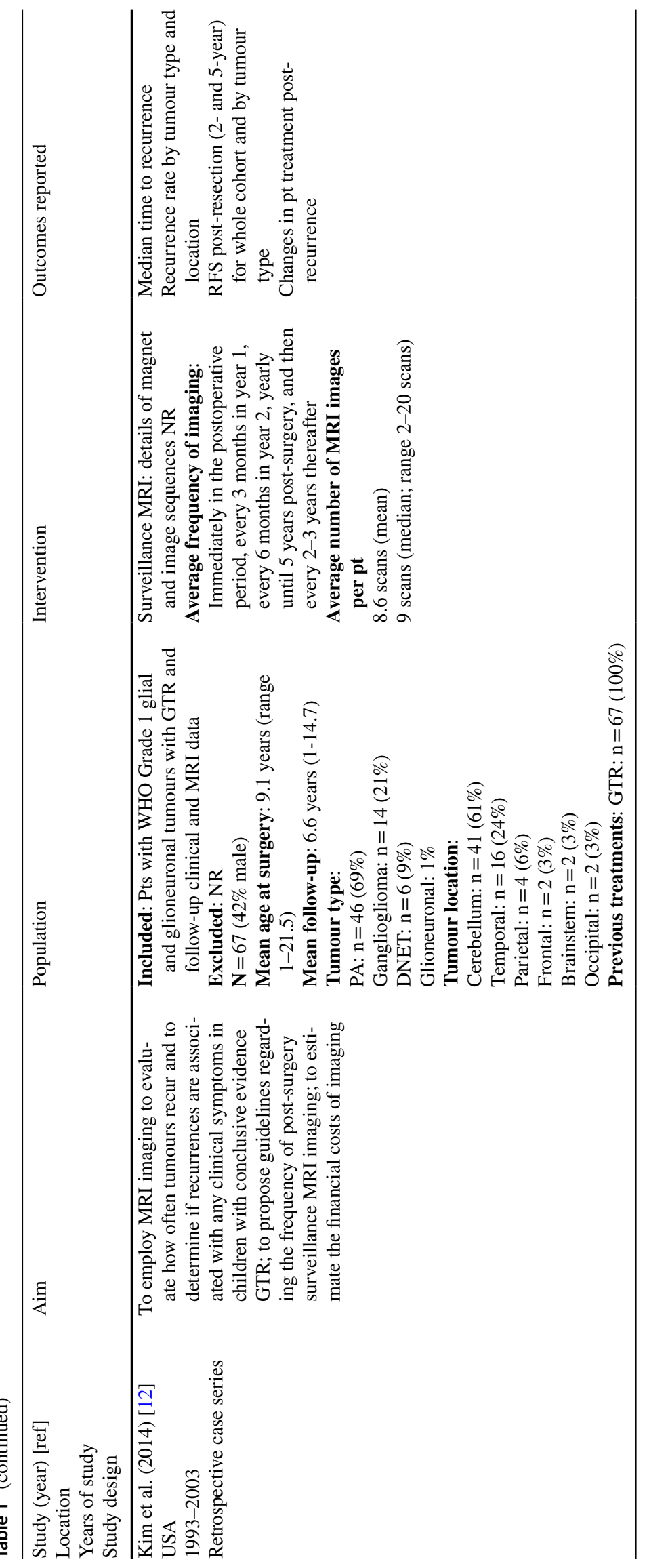




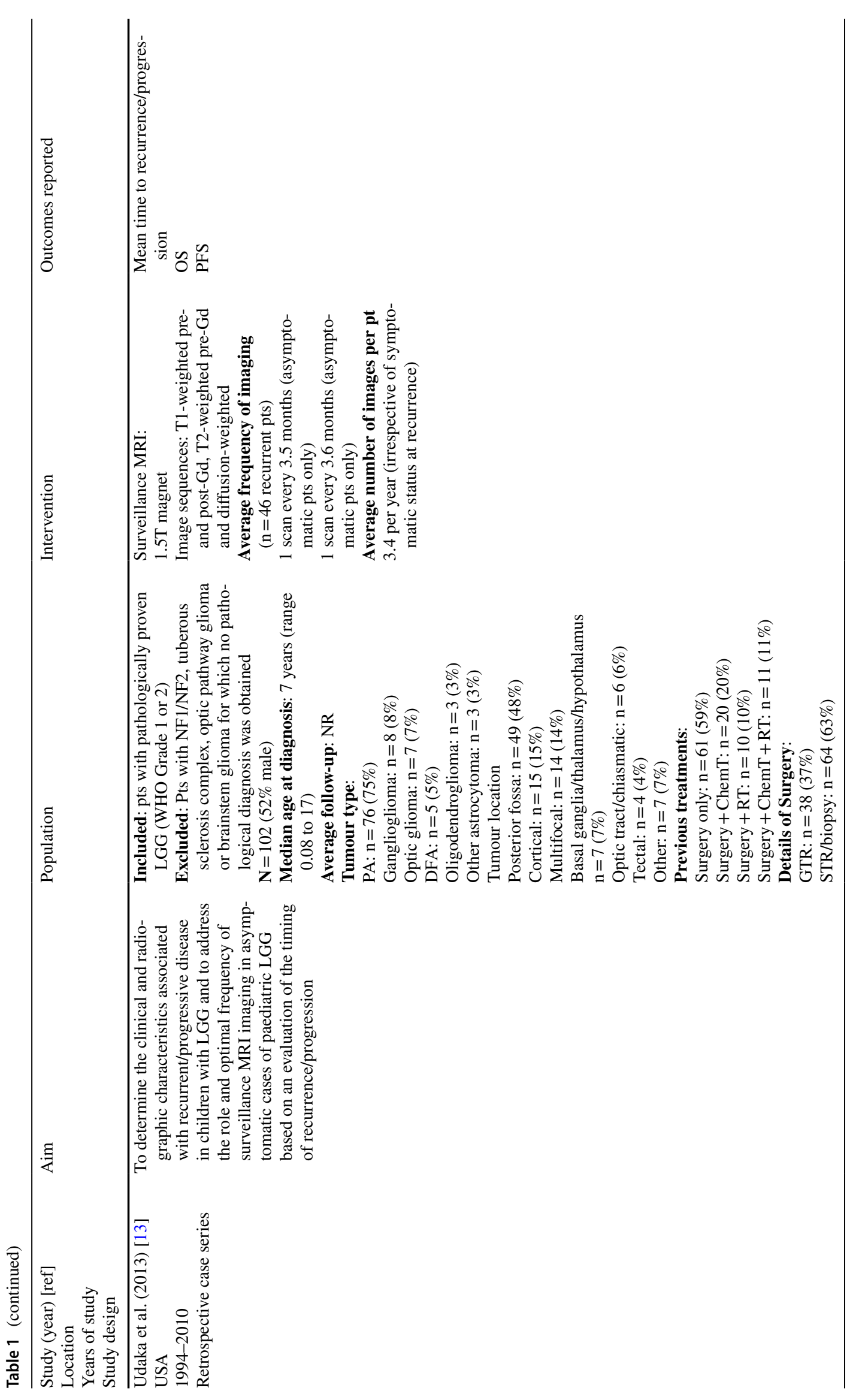




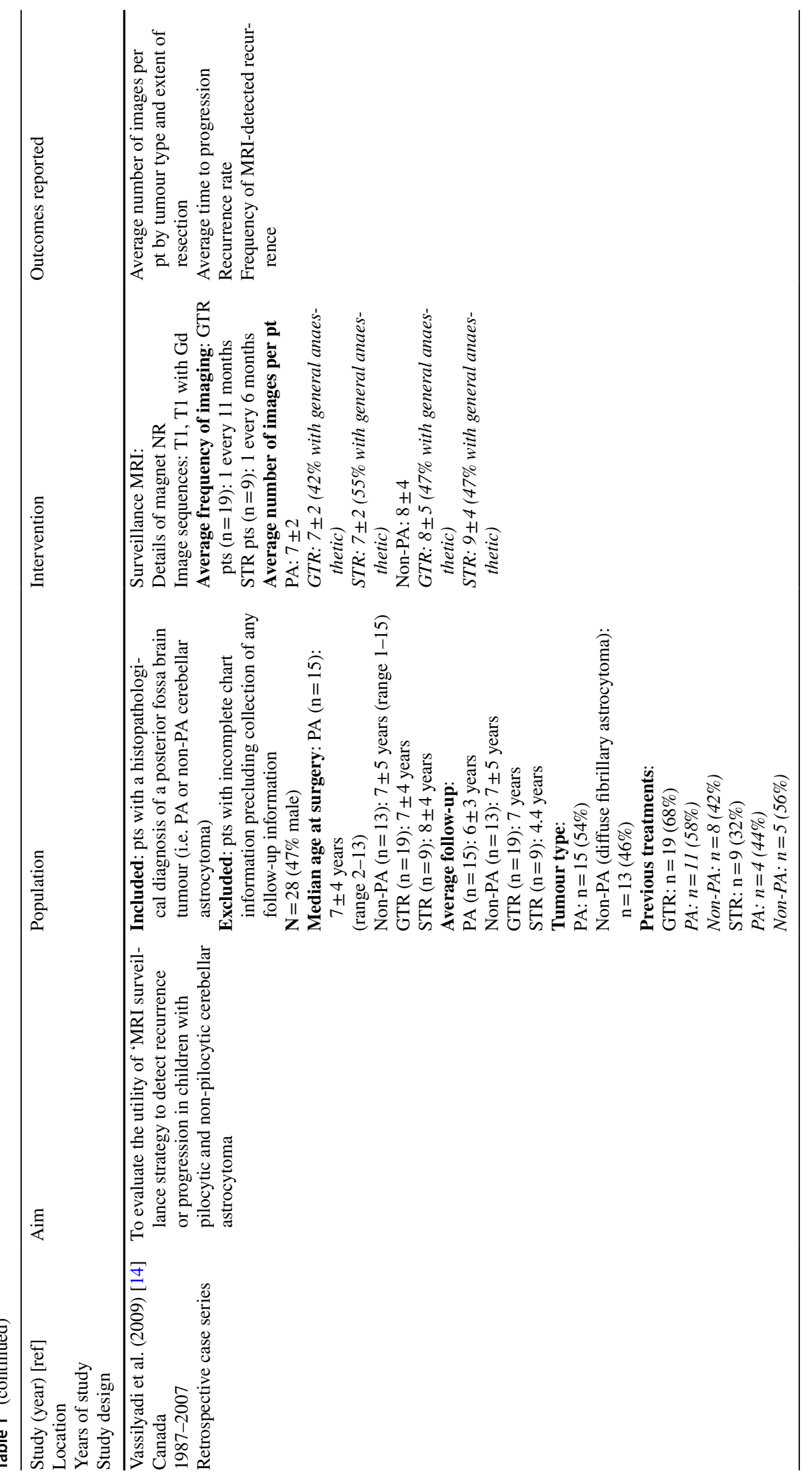




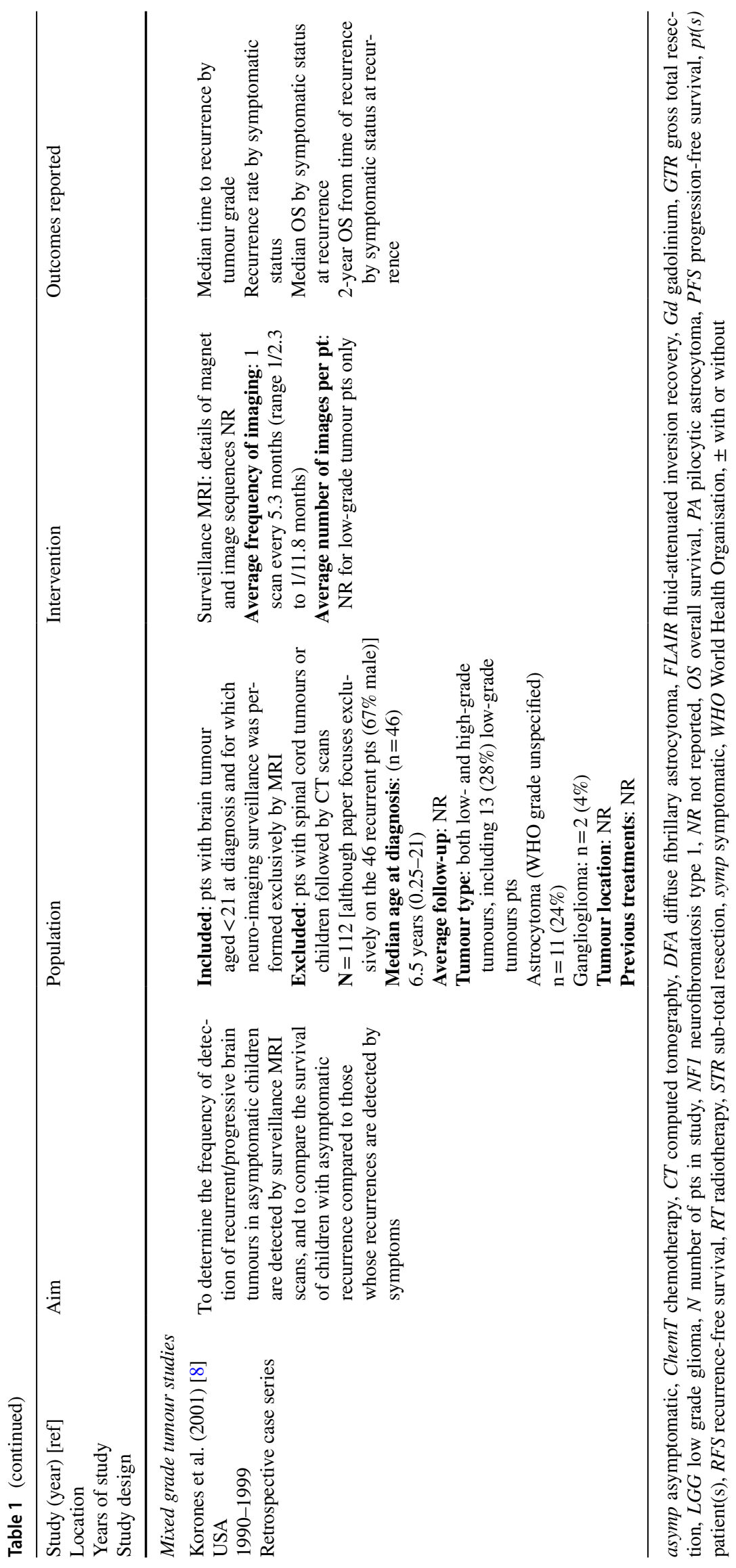




\section{Imaging schedules/frequency of imaging}

Whilst the reporting of the frequency of scanning varied between studies, with some [9-11] reporting scanning intervals and others $[9,11,12]$ reporting the number of scans per patient, a discernible pattern emerged whereby imaging was at its most frequent within the first year (2-4 follow-up scans), reducing to around half this frequency in year two and then becoming annual thereafter for the duration of follow-up. Vassilyadi et al. [14] reported frequency in terms of scanning intervals by extent of resection, with STR patients undergoing almost twice as many scans as GTR patients (one scan every 6 months vs 11 months respectively) while Udaka et al. [13] reported intervals with respect to recurrent patients only ( 1 scan every 3.5 months) rather than the total number of patients at the beginning of the study.

Five studies reported the average number of MRI scans per patient for the duration of follow-up [9, 11-14], ranging from six [11] to 17 scans [13]. This however is somewhat misleading as studies were inconsistent in terms of reporting as Udaka et al. [13] reported the number of scans for recurrent patients only, Alford et al. [9] the average number of scans for GTR patients (median nine) and Dodgshun et al. [10] stated the recommendations pertaining at their institution at the time of publication (nine scans in the first 5 years).

Vassilyadi et al. [14] reported the average number of scans by tumour sub-group with the median for PA patients comparable to that for non-PA patients (seven vs eight/nine respectively). The distribution of scans over the follow-up period was not reported.

\section{Rates of recurrence}

Overall, of the total of 357 patients, 98 cases (24\%) of recurrence occurred. Recurrence rates by study ranged from 5 to $41 \%$ of patients. Five studies reported recurrence by symptomatic status at recurrence, of which $0-35 \%$ of patients were symptomatic while $65-100 \%$ of patients were asymptomatic [10-14]. (See Table 2).

\section{Recurrence rates by tumour type}

Of 297 patients with PA, 70 (24\%) recurred with recurrence rates across the six studies ranging from 5\% [10] to $47 \%$ [13]. Of 22 ganglioglioma [12, 13], six DNET [12] and 13 non-PA/DFA [14] patients, five, one and one patients recurred respectively. Udaka, the only study to report both first and subsequent recurrences, reported eight recurrences across three unspecified PA patients [13]. (See Table 3).

Asymptomatic recurrence rates for PA were calculable in four studies, ranging from 82 to $100 \%$ [10-12, 14]. Across the four studies, $96 \%$ of recurrences were asymptomatic.
Kim reported that the three recurrences of ganglioglioma and one recurrence of DNET were all asymptomatic at recurrence [12]. The patient with progressive non-PA was asymptomatic at recurrence [14].

There were no recurrences observed in patients with glioneuronal tumour, optic glioma and oligodendroglioma although the number of patients with each of these tumour types was so small that no inferences should be drawn regarding recurrence in these patients.

\section{Recurrence rates by tumour site}

While most studies reported patients by both tumour type and location, most outcomes, including recurrence, were reported by tumour type alone and therefore it was not possible to discern the effect of tumour location on recurrence. Only Dodgshun et al. [10], with nine of 67 PAs located supratentorially, reported that there was "no difference in recurrence rate with regard to tumour site $(p=0.37)$.'

\section{Recurrence rates by extent of resection}

The three studies consisting solely of GTR patients reported recurrence rates of $4 \%(n=3 / 67), 28 \%(n=11 / 40)$ and $19 \%$ $(n=13 / 67)$ respectively [10-12]. Three of the four studies with both GTR and STR patients reported recurrence rates for GTR patients of $15 \%(n=6 / 41), 24 \%(n=9 / 38)$ and $0 \%(n=0 / 19)$ respectively and rates of recurrence for STR patients of $33 \%(n=4 / 12), 55 \%(n=35 / 64)$ and $22 \%$ $(n=2 / 9)$ respectively $[9,13,14]$ (See Table 4$)$.

\section{Diagnostic yield of imaging}

The diagnostic yield of MRI, or the number of scans identifying recurrence as a proportion of total scans, was reported in three studies $[9,10,14]$. For Alford, diagnostic yield was $2 \%$ (i.e. one recurrence detected every 50 scans) based on 6 of 41 predominantly GTR patients [9]. As the symptomatic status of recurrent patients was not reported, diagnostic yield by symptomatic status was not calculable. For Dodgshun, diagnostic yield was $0.25 \%$ (one asymptomatic recurrence detected with 399 scans) based on 33 patients with at least 5 years follow-up [10]. For Vassilyadi, diagnostic yield was $1 \%$ (two asymptomatic recurrences detected with 216 scans) in STR patients [14].

\section{Time to recurrence}

Five studies [8, 10-14] reported average time to recurrence post-primary treatment ranging from 0.33 [14] to 2.33 years [8]. (See Table 2). In two studies, recurrence was $100 \%$ asymptomatic with median times to recurrence of 1.9 and 


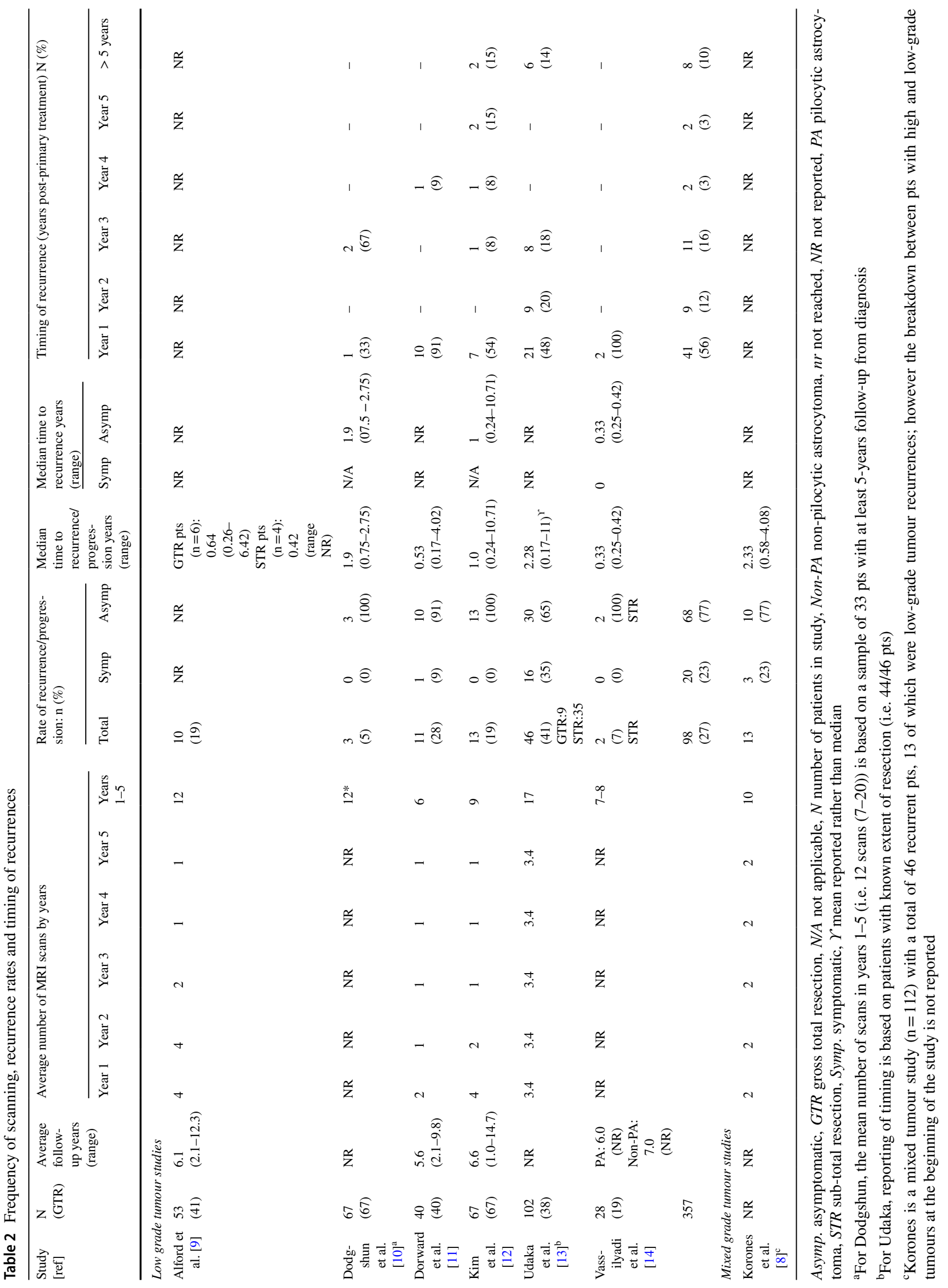


Table 3 Recurrence rates and timing of recurrence by tumour type

\begin{tabular}{|c|c|c|c|c|c|c|c|}
\hline \multirow[t]{2}{*}{ Study [ref] } & \multirow[t]{2}{*}{$\mathrm{N}$ of pts } & \multicolumn{3}{|c|}{$\begin{array}{l}\text { Patients with recurrent/progressive } \\
\text { disease: } \mathrm{n}(\%)\end{array}$} & \multirow[t]{2}{*}{$\begin{array}{l}\text { Median time to recurrence: } \\
\text { years (range) }\end{array}$} & \multicolumn{2}{|c|}{$\begin{array}{l}\text { Median time to recur- } \\
\text { rence: } \\
\text { years (range) }\end{array}$} \\
\hline & & Total & Symp & Asympt & & Sympt & Asympt \\
\hline \multicolumn{8}{|c|}{ (a) Low-grade tumour studies } \\
\hline \multicolumn{8}{|l|}{ Pilocytic astrocytoma } \\
\hline Alford et al. [9] & 53 & $10(19)$ & NR & NR & $\begin{array}{l}\text { GTR pts }(n=6) \\
0.64(0.26-6.42) \\
\text { STR pts }(n=4) \\
5.23 \text { (range NR) }\end{array}$ & NR & NR \\
\hline Dodgshun et al. [10] & 67 & $3(5)$ & $0(0)$ & $3(100)$ & $1.9(0.75-2.75)$ & 0 & $\begin{array}{l}1.9 \\
(07.5-2.75)\end{array}$ \\
\hline Dorward et al. [11] & 40 & $11(28)$ & $1(9)$ & $10(91)$ & $0.53(0.17-4.02)$ & NR & NR \\
\hline Kim et al. [12] & 46 & $9(20)$ & $0(0)$ & $9(100)$ & NR & NR & NR \\
\hline Udaka et al. [13] & 76 & $36(47)$ & NR & NR & NR & NR & NR \\
\hline Vassilyadi et al. [14] & 15 & $1(7)$ & $0(0)$ & $1(100)$ & 0.25 & 0 & 0.25 \\
\hline Totals & 297 & $70(24)$ & $1(4)$ & $23(96)$ & & & \\
\hline \multicolumn{8}{|c|}{ Diffuse fibrillary astrocytoma } \\
\hline Vassilyadi et al. [14] & 13 & $1(8)$ & $0(0)$ & $1(100)$ & 0.42 & 0 & 0.42 \\
\hline \multicolumn{8}{|l|}{$\begin{array}{l}\text { Other astrocytoma (WHO } \\
\text { grade not specified) }\end{array}$} \\
\hline Udaka et al. [13] & 3 & $8(267)$ & NR & NR & NR & NR & NR \\
\hline \multicolumn{8}{|l|}{ Ganglioglioma } \\
\hline Kim et al. [12] & 14 & $3(21)$ & 0 & $3(100)$ & NR & NR & NR \\
\hline Udaka et al. [13] & 8 & $2(25)$ & NR & NR & NR & NR & NR \\
\hline Totals & 22 & $5(23)$ & 0 & $3(100)$ & & & \\
\hline \multicolumn{8}{|c|}{ Dysembryoplastic neuroepithelial tumours (DNET) } \\
\hline Kim et al. [12] & 6 & $1(17)$ & 0 & $1(100)$ & NR & NR & NR \\
\hline \multicolumn{8}{|c|}{ (b) Mixed-grade tumour study (Korones) } \\
\hline $\begin{array}{l}\text { Other astrocytoma (WHO } \\
\text { grade not specified) }\end{array}$ & & $11^{\mathrm{a}}$ & $2(18)$ & $9(82)$ & NR & NR & NR \\
\hline Ganglioglioma & & 2 & $1(50)$ & $1(50)$ & NR & NR & NR \\
\hline
\end{tabular}

Asymp. asymptomatic, GTR gross total resection, $N$ number of patients in study, $N R$ not reported, STR sub-total resection, Symp. symptomatic

${ }^{a}$ Korones et al. [8] was the only study which did not provide a breakdown of the patients at the beginning of the study in terms of tumour type and, as such, the number of recurrences in this study $(n=13)$ has not been taken into account when calculating the percentage of the total number of patients at baseline with each tumour type which went on to experience a recurrence

1.0 years respectively $[10,12]$. Neither of the studies containing mixed low-grade tumour types reported median time to recurrence by tumour type $[12,13]$.

\section{Time to recurrence by extent of resection}

Three studies with exclusively GTR patients reported median times to recurrence of 0.53 [11], 1.0 [12] and 1.9 [10] years respectively. Of the three studies containing both GTR and STR patients, two reported median times to recurrence of 2.28 [13] and 0.33 years [14] respectively. Only Alford et al. [9] reported median time to recurrence solely by extent of resection [GTR 0.64 vs STR 0.42 years respectively $(\mathrm{p}<0.0001)]$.

\section{Timing of recurrences}

Five studies provided data on the timing of recurrences post-diagnosis/primary treatment [10-14]. Collectively, $56 \%$ of recurrences took place within the first year postprimary treatment (with $46 \%$ of these within the first six months), $68 \%$ by year two and $90 \%$ by year five. Neither of the studies with mixed tumour populations reported timing of recurrences by tumour type $[12,13]$.

\section{Patient management post-recurrence}

Four studies reported details of patient management following recurrence with respect to 29 patients, 28 of whom 
Table 4 Breakdown of lowgrade tumour patients by extent of resection

\begin{tabular}{|c|c|c|c|c|c|c|c|}
\hline \multirow[t]{2}{*}{ Author (year) [ref] } & \multirow[t]{2}{*}{$\mathrm{N}$} & \multirow[t]{2}{*}{$\begin{array}{l}\text { Rec } \\
N\end{array}$} & \multicolumn{2}{|l|}{$\begin{array}{l}\text { GTR } \\
\text { n (\%) }\end{array}$} & \multicolumn{2}{|l|}{$\begin{array}{l}\text { STR } \\
\text { n (\%) }\end{array}$} & \multirow[t]{2}{*}{ N/A } \\
\hline & & & All pts & Rec pts & All pts & Rec pts & \\
\hline \multicolumn{8}{|l|}{ GTR pts only } \\
\hline Dodgshun et al. (2016) [10] & 67 & 3 & $67(100)$ & $3(4)$ & N/A & N/A & N/A \\
\hline Dorward et al. (2010) [11] & 40 & 11 & $40(100)$ & $11(28)$ & N/A & N/A & N/A \\
\hline Kim et al. (2014) [12] & 67 & 13 & $67(100)$ & $13(19)$ & N/A & N/A & N/A \\
\hline \multicolumn{8}{|l|}{ GTR and STR pts } \\
\hline Alford et al. (2016) [9] & 53 & 10 & $41^{\mathrm{a}}(77)$ & $6(15)$ & $12(23)$ & $4(33)$ & N/A \\
\hline Korones et al. (2014) [8] & NR & - & NR & NR & NR & NR & NR \\
\hline Udaka et al. (2013) [13] & 102 & 46 & $38(37)$ & $9(24)$ & $64(63)$ & $35(55)$ & $2(4)$ \\
\hline Vassilyadi et al. (2009) [14] & 28 & 2 & $19(68)$ & $0(0)$ & $9(32)$ & $2(22)$ & N/A \\
\hline Totals & 357 & 85 & $272(76)$ & $42(15)$ & $85(24)$ & $41(48)$ & $2(3)$ \\
\hline
\end{tabular}

GTR gross total resection, N/A not applicable, Pts patients, $N$ number of patients in study, $N$ total number of patients in study, NR not reported, NR not reported, Rec $n$ number of recurrent patients, STR sub-total resection

${ }^{\mathrm{a}} \mathrm{GTR} /$ indeterminate were asymptomatic [10-12, 14]. Twenty-six patients underwent a change in their management including repeat surgery $(n=21)$, chemotherapy $(n=2)$, radiotherapy $(n=2)$ and radiosurgery $(n=1)$. The remaining three patients were observed.

\section{Survival}

All but two studies reported some type of survival outcome $[9,14]$.

\section{Overall survival (OS)}

Four studies reported OS up until the time of reporting [10-13]. In two studies of PA [10,11] OS was $100 \%$ and in the two mixed tumour studies $[12,13]$ OS was 96 and $100 \%$ respectively, all measured from recurrence.

\section{Surrogate survival outcomes}

Four studies reported surrogate survival outcomes [10-13]. Dodgshun et al. [10] reported 5-year EFS of 95\% (95\% CI 90-100\%). Dorward reported RFS by evidence of 'nodular enhancement' on surveillance MRI within the first 3-6 months, with both the 5- and 10-year RFS for PA patients whose scans lacked evidence of nodular enhancement of $90 \%$ compared to patients whose scans evidenced nodular enhancement of 44 and $22 \%$ respectively [11]. Kim reported 2- and 5-year RFS for 67 patients of $90 \%$ and $82 \%$ respectively, as well as RFS by tumour type, with 2- and 5-year RFS for PA, ganglioglioma and DNET of 87 and 82, 93 and 85 and 100 and $67 \%$ respectively [12].
Udaka reported median PFS for all 102 patients (4.7 years) and separately for patients with PA (4.2 years) who represented $75 \%$ of the study population [13]. Median PFS for GTR patients was significantly greater than STR/biopsy patients [not reached versus 2.1 years respectively $(\mathrm{p}=0.012)$ ]. Udaka also found that while recurrence was reduced in GTR patients, it occurred earlier compared to those with less than total resection (16.6 vs 25.8 months, respectively).

\section{Quality of survival}

None of the studies reported quality of survival of the children and their families.

\section{Discussion}

This systematic review was borne out of discussions between the project team and the PPI group, which consisted largely of mothers of children with CNS tumours. Of particular interest to the PPI group was surveillance scanning and its frequency. They remarked that scanning was a significant and time-consuming part of their lives and a major source of anxiety to the whole family before, during and after each scan. However, they were unanimous that scanning was something they were prepared to endure so long as this was an evidence-based practice that ultimately benefitted their children. They were surprised to learn that, despite being routine practice, there are no internationally adopted guidelines for the frequency and duration of surveillance MRI in paediatric CNS tumours. This issue is not only important to patients and their families but also to health care systems such as the NHS in terms of direct and indirect healthcare 
costs. A surveillance imaging programme needs to detect recurrent disease ahead of onset of signs/symptoms and to result in changes in patient management which bestow a long-term clinical benefit in terms of improved patient outcomes (i.e. reduced mortality and/or improved quality of survival). Ultimately, the assessment of both the benefits and costs of this practice should be based on research evidence and this is what prompted us to undertake the current review.

Six low-grade tumour surveillance imaging studies were excluded from the review as they employed both CT and MRI as surveillance imaging modalities but did not report results separately by modality [15-20]. No comparative studies assessing the effectiveness of routine surveillance screening with MRI were identified. The evidence base to guide the clinical practice of surveillance MRI for children with low-grade CNS tumours consisted of seven small retrospective, single arm observational studies in which dataacquired for clinical purposes rather than assessment of surveillance imaging protocols - was analysed to determine the optimal frequency and/or duration of surveillance MRI. Six of these studies consisted solely of patients $(n=357)$ with low-grade CNS tumours while one study, comprising a mixture of low- and high-grade tumour patients $(n=112)$, reported on 13 low-grade tumour patients [8]. In all studies, MRI was employed exclusively as the imaging modality with all of the patients having undergone surgery as a primary treatment, achieving either GTR or STR. For all studies, both the number and rates of recurrence were low, with the majority of recurrences diagnosed asymptomatically via surveillance MRI and tending to occur within the first 2 years following primary treatment, suggesting there may be scope for reducing the number and frequency of later scans (10\% of recurrences occurred post-five years, although patient characteristics of these individuals were not described). The extent of initial resection also appeared to be associated with recurrence, with patients achieving GTR significantly less likely to experience recurrence.

Although all seven studies reported essentially similar results, study authors differed in their interpretation, leading to opposing conclusions regarding the optimal frequency and/or duration of surveillance with respect to GTR patients (see Online Resource 6). For example, Alford et al. [9] concluded that frequent imaging of GTR patients may be unwarranted beyond the radiological confirmation of GTR documented on two consecutive scans separated by at least 3 months; likewise the study by Vassilyadi et al. [14] concluded that GTR patients may not benefit from surveillance, although this was based solely on recurrences in two STR patients. Conversely, Udaka et al. [13] advised caution, recommending surveillance up to 5 years, irrespective of the extent of resection. Similarly Dorward, despite identifying associations predictive of recurrence in GTR patients $(\mathrm{p}<0.05)$, also erred on the side of caution, albeit based on limited data (i.e. one delayed recurrence) [11]. Both Dodgshun et al. [10] and Kim et al. [12], based on the timing of recurrences post-diagnosis, suggested reduced imaging schedules but argued that long-term imaging (5 and 10 years respectively) for GTR patients was necessary although, again, this was based on a very small number of recurrences-three and 13 recurrences respectively. Overall, it is interesting to note that all of these conclusions and recommendations were based on low recurrence numbers, ranging from 0 [14] to 13 [12].

As demonstrated by the study authors, drawing conclusions from these studies is problematic. The potential for bias with case series studies is considerable making any conclusions from this review highly tentative and to be viewed with extreme caution. For instance, patient populations across the studies were highly selected with the main patient group being children with posterior fossa PA that had been completely resected. Half the studies excluded patients with low-grade tumour predisposition syndromes such as NF1 and tuberous sclerosis [9, 11, 13]. One study [13] differed from the others by including a large number of patients with low grade gliomas at all sites who had immediate adjuvant therapy post-surgery, making them a population with a significantly higher risk of recurrence. All of this selection bias is likely to skew the results of this review. The review question needs to be properly investigated within an RCT. Of particular importance in paediatric low-grade tumour studies, where survival is generally excellent, patient-reported quality of survival should be a priority: none of the studies reported this outcome. Future trials should also examine potential adverse events resulting from the repeated administration of contrast materials (e.g. Gadolinium [21]) and in younger children, anaesthesia and sedatives. Although an RCT would be challenging to design and conduct, the results of this review demonstrate that we are at equipoise as to the optimum scanning regimen. Scanning is a vital part of the treatment pathway for children with CNS tumours and has the potential to improve survival but also has risks associated with it. Its optimum use therefore needs to be established.

To conclude, despite the existence of various consensus recommendations $[22,23]$, this systematic review did not identify any studies that were able to inform best practice as to the frequency or duration of surveillance MRI in asymptomatic children with LGG. The findings could however inform the development of future clinical trials, particularly regarding scanning frequency and duration.

Acknowledgements We would like to acknowledge the input of the Patient and Public Involvement (PPI) group and the wider clinical team who helped to frame the review question and contribute to the direction of the paper. 
Author contributions SPS conceived and designed the study and wrote the article. CM conceived and designed the study and read and commented on the article. SB conceived the study concept, provided clinical input and read and commented on the article. BP conceived the study, provided clinical input and read and commented on the article. ME conceived the study, provided clinical input and read and commented on the article. RP conceived the study, provided clinical and methodological input and read and commented on the article. AP provided clinical input and read and commented on the article. SA provided clinical input and read and commented on the article. SW provided clinical input and read and commented on the article. KW conceived and designed the study, provided methodological and statistical input and read and commented on the article. PRK provided clinical input and read and commented on the article. JSW conceived and designed the study, revised the article and is the guarantor of the review. All authors read and approved the final manuscript.

Funding This paper presents independent research funded by the National Institute for Health Research (NIHR) under its Research for Patient Benefit (RfPB) Programme (Grant Reference Number PB-PG1112-29122). Pam Kearns, Keith Wheatley and Jayne Wilson received funding from Cancer Research UK. AP is funded by an NIHR Research Professorship (13-0053). The views expressed are those of the author(s) and not necessarily those of the NHS, the NIHR, the Department of Health or Cancer Research UK.

\section{Compliance with ethical standards}

Conflict of interest The authors declare that they have no conflict of interest.

Ethical approval For this type of study (i.e. retrospective), formal consent is not required.

Informed consent For this type of study i.e. systematic review, informed consent was not required.

Research involving human participants and/or animals This article does not contain any studies with human participants or animals performed by any of the authors.

Open Access This article is distributed under the terms of the Creative Commons Attribution 4.0 International License (http://creativeco mmons.org/licenses/by/4.0/), which permits unrestricted use, distribution, and reproduction in any medium, provided you give appropriate credit to the original author(s) and the source, provide a link to the Creative Commons license, and indicate if changes were made.

\section{References}

1. Sievert AJ, Fisher MJ (2009) Pediatric low-grade gliomas. J Child Neurol 24(11):1397-1408. https://doi.org/10.1177/08830 73809342005

2. Elterman RD, Bruce DA (1999) The continued surveillance for recurrent medulloblastoma/primitive neuro-ectodermal tumor (Abstract). Pediatr Neurosurg 19:322

3. Kramer ED, Vezina LG, Packer RJ, Fitz CR, Zimmerman RA, Cohen MD (1994) Staging and surveillance of children with central nervous system neoplasms: recommendations of the neurology and tumor imaging committees of the children's cancer group. Pediatr Neurosurg 20:254-263. https://doi. org/10.1159/000120799

4. Jayaraman M (2009) Adult brain tumors. In: Atlas SW (ed) Magnetic resonance imaging of the brain and spine, 4th edn. Lippincott Williams \& Wilkins, Philadelphia, PA, pp 445-590

5. Moher D, Liberati A, Tetzlaff J, Altman DG (2009) Preferred reporting items for systematic reviews and meta-analyses: the PRISMA statement. Ann Intern Med 151(4):264-269. https:// doi.org/10.1136/bmj.b2535

6. Main C, Stevens SP, Bailey S, Phillips R, Pizer B, Wheatley K et al (2016) The impact of routine surveillance screening with magnetic resonance imaging (MRI) to detect tumour recurrence in children with central nervous system (CNS) tumours: protocol for a systematic review and meta-analysis. Syst Rev 5(1):143. https:// doi.org/10.1186/s13643-016-0318-1

7. Centre for Reviews and Dissemination (CRD) UoY (2008) Systematic reviews: CRD's guidance for undertaking reviews in health care. Published by CRD, University of York

8. Korones DN, Butterfield R, Meyers SP, Constine LS (2001) The role of surveillance magnetic resonance imaging (MRI) scanning in detecting recurrent brain tumors in asymptomatic children. $\mathbf{J}$ Neurooncol 53(1):33-38. https://doi.org/10.1023/A:101180440

9. Alford R, Gargan L, Bowers DC, Klesse LJ, Weprin B, Koral K (2016) Postoperative surveillance of pediatric cerebellar pilocytic astrocytoma. J Neurooncol (2016) 130:149-154. https://doi. org/10.1007/s11060-016-2222-x

10. Dodgshun AJ, Maixner WJ, Hansford JR, Sullivan MJ (2016) Low rates of recurrence and slow progression of pediatric pilocytic astrocytoma after gross-total resection: justification for reducing surveillance imaging. J Neurosurg Pediatr 17(5):569-572. https ://doi.org/10.3171/2015.9.PEDS15449

11. Dorward IG, Luo J, Perry A, Gutmann DH, Mansur DB, Rubin JB et al (2010) Postoperative imaging surveillance in pediatric pilocytic astrocytomas. J Neurosurg Pediatr 6(4):346-352. https ://doi.org/10.3171/2010.7.PEDS10129

12. Kim AH, Thompson EA, Governale LS, Santa C, Cahll K, Kieran MW et al (2014) Recurrence after gross-total resection of lowgrade pediatric brain tumors: the frequency and timing of postoperative imaging. J Neurosurg Pediatr 14(4):356-364. https://doi. org/10.3171/2014.6.PEDS1321

13. Udaka YT, Yeh-Nayre LA, Amene CS, VandenBerg SR, Levy ML, Crawford JR (2013) Recurrent pediatric central nervous system low-grade gliomas: the role of surveillance neuroimaging in asymptomatic children. J Neurosurg Pediatr 11(2):119-126. https ://doi.org/10.3171/2012.10.PEDS12307

14. Vassilyadi M, Shamji MF, Tataryn Z, Keene D, Ventureyra E (2009) Postoperative surveillance magnetic resonance imaging for cerebellar astrocytoma. Can J Neurol Sci 36(6):707-712. https:// doi.org/10.1017/S0317167100008313

15. Hirpara DH, Bhatt MD, Katrin S (2016) Utility of long-term surveillance neuroimaging five years post diagnosis in the management of pediatric brain tumours. Austin Pediatr Oncol 1(1):1002. http://austinpublishinggroup.com/pediatric-oncology/fulltext/ apo-v1-id1002.php

16. Kovanlikaya A, Karabay N, Çakmakçi H, Uysal K, Olgun N, Ergör G (2003) Surveillance imaging and cost effectivity in pediatric brain tumors. Eur J Radiol 47(3):188-192. https://doi. org/10.1016/S0720-048X(02)00213-9

17. Ogiwara H, Bowman RM, Tomita T (2012) Long-term followup of pediatric benign cerebellar astrocytomas. Neurosurgery 70(1):40-47. https://doi.org/10.1227/NEU.0b013e31822ff0ed

18. Steinbok P, Hentschel S, Cochrane DD, Kestle JR (1996) Value of postoperative surveillance imaging in the management of children with some common brain tumors. J Neurosurg 84(5):726-732. https://doi.org/10.1007/978-3-7091-6090-9_4 
19. Saunders DE, Phipps KP, Wade AM, Hayward RD (2005) Surveillance imaging strategies following surgery and/or radiotherapy for childhood cerebellar low-grade astrocytoma. J Neurosurg 102(2 Suppl):172-178. https://doi.org/10.3171/jns.2005.102.2.0172

20. Sutton LN, Cnaan A, Klatt L, Zhao H, Zimmerman R, Needle M et al (1996) Postoperative surveillance imaging in children with cerebellar astrocytomas. J Neurosurg 84(5):721-725. https://doi. org/10.3171/jns.1996.84.5.0721

21. Khawaja AZ, Cassidy DB, Al Shakarchi J, McGrogan DG, Inston NG, Jones RG (2015) Revisiting the risks of MRI with
Gadolinium based contrast agents-review of literature and guidelines. Insights Imaging 6(5):553-558. https://doi.org/10.1007/ s13244-015-0420-2

22. Ellingson BM, Bendszus M, Boxerman J, Barboriak D, Erickson BJ, Smits M et al (2015) Consensus recommendations for a standardized brain tumor imaging protocol in clinical trials. Neuro Oncol 17(9):1188-1198. https://doi.org/10.1093/neuonc/nov095

23. Craig E, Connolly DJ, Griffiths PD, Raghavan A, Lee V, Batty R (2015) MRI protocols for imaging paediatric brain tumours. Clin Radiol 67(9):829-832. https://doi.org/10.1016/j.crad.2012.03.018 